Pacific Journal of Mathematics

FREE PRODUCTS OF TOPOLOGICAL GROUPS WITH 


\title{
FREE PRODUCTS OF TOPOLOGICAL GROUPS WITH AMALGAMATION
}

\author{
Elyahu Katz and Sidney A. Morris
}

\begin{abstract}
It is proved that the free product of any two $k_{\omega}$-groups with a compact subgroup amalgamated is a $k_{\omega}$-group, and in particular, Hausdorff.
\end{abstract}

1. Introduction. In recent years much work has been done on describing the topology of free products of topological groups (see for example $[1,3,8,10,12,13,15])$. From there it is natural to progress to free products with amalgamation.

One would hope that the free product with amalgamation of any Hausdorff topological groups exists, is Hausdorff and its underlying group structure is the amalgamated free product of the underlying groups. This would include as a special case Graev's theorem [2] that the free product of Hausdorff groups is Hausdorff. As his proof is certainly non-trivial, it should not be expected that this "hope" will be easily verified, even if the result is true.

The first contribution to this problem was by Ordman [13], who showed that the amalgamated free product of certain locally invariant Hausdorff topological groups is Hausdorff. The next contribution was by Khan and Morris [5] who proved the Hausdorffness of the free product of Hausdorff groups with a central subgroup amalgamated. This has recently been extended by Katz and Morris [4] to free products of $k_{\omega}$-groups with a closed normal subgroup amalgamated.

Most of the work on free topological groups and free products of topological groups in fact deals with topological groups which are $k_{\omega^{-}}$ spaces. Therefore, the result we would like to have is that the amalgamated free product of $k_{\omega}$-groups is a $k_{\omega}$-group. This would imply La Martin's theorem that epics in the category of $k_{\omega}$-groups have dense range. (See [6], [11] and [14].) We prove here that the free product of any two $k_{\omega}$-groups with a compact subgroup amalgamated is a $k_{\omega}$-group and in particular Hausdorff. This result includes a large class of examples not covered by [4] or [13], since every connected locally compact Hausdorff topological group, $G$ is a $k_{\omega}$-group and has a compact subgroup $K$ such 
that $G$ is homeomorphic to $\mathbf{R}^{n} \times K$, for some Euclidean group $\mathbf{R}^{n}$. Such examples with $G$ not locally invariant and $K$ not normal exist in profusion.

2. Definitions and statements of the main result. The standard references for amalgamated free products of groups are B. H. Neumann [9] and Magnus, Karrass and Solitar [7]. For completeness we include some definitions here.

Definition. Let $A$ be a common subgroup of groups $G$ and $H$. The group $G *{ }_{A} H$ is said to be the free product of $G$ and $H$ with amalgamated subgroup $A$ if

(i) $G$ and $H$ are subgroups of $G *{ }_{A} H$,

(ii) $G \cup H$ generates $G *{ }_{A} H$ algebraically,

(iii) every pair $\phi_{1}, \phi_{2}$ of homomorphisms of $G$ and $H$, respectively, into any group $D$ which agree on $A$, extend to a homomorphism $\Phi$ of $G *{ }_{A} H$ into $D$.

DeFinition. Let $A$ be a common subgroup of topological groups $G$ and $H$. The topological group $G *{ }_{A} H$ is said to be the free product of the topological groups $G$ and $H$ with amalgamated subgroup $A$ if

(i) $G$ and $H$ are topological subgroups of $G *{ }_{A} H$,

(ii) $G \cup H$ generates $G *{ }_{A} H$ algebraically, and

(iii) every pair $\phi_{1}, \phi_{2}$ of continuous homomorphisms of $G$ and $H$, respectively, into any topological group $D$, which agree on $A$, extend to a continuous homomorphism of $G *{ }_{A} H$ into $D$.

Our main result is the following:

THEOREM. Let $F$ and $G$ be $k_{\omega}$-groups with a common compact subgroup $A$. Then the free product of $F$ and $G$ with $A$ amalgamated is a $k_{\omega}$-group. (In particular, the amalgamated free product $F *{ }_{A} G$ is Hausdorff.)

3. Yoffi representations. To prove the main theorem it suffices to show that the kernel, $K$, of the canonical homomorphism $\Phi: F * G \rightarrow$ $F *{ }_{A} G$ is closed. The first step in doing this is to find a nice representation for the elemnts of $K$. This will be achieved in Proposition 1.

Notation. We denote the embedding map of $A$ in $F$ by $f$ and the embedding map of $A$ in $G$ by $g$.

It is readily seen that $K$ is the normal subgroup generated by $\left\{f(a) g(a)^{-1}: a \in A\right\}$. Further, observe that each $w \in K$ has a representation. 
$(*) \quad\left\{\begin{array}{c}w=\left(t_{1,1} \cdots t_{1, q_{1}} v_{1} t_{1, q_{1}}^{-1} \cdots t_{1,1}^{-1}\right)\left(t_{2,1} \cdots t_{2, q_{2}} v_{2} t_{2, q_{2}}^{-1} \cdots t_{2,1}^{-1}\right) \\ \cdots\left(t_{s, 1} \cdots t_{s, q_{s}} v_{s} t_{s, q_{s}}^{-1} \cdots t_{s, 1}^{-1}\right),\end{array}\right.$

where for each $j, t_{j, l}$ lies in $F$ or $G$, and $t_{j, l}$ and $t_{j, i+1}$ do not both belong to $F$ or both belong to $G$, and each $v_{i} \in\left\{f(a) g(a)^{-1}, g(a) f(a)^{-1}: a \in A\right\}$.

DeFinitions. For each representation of the form $(*)$ we define the weight of the representation to be a sequence $\left(a_{1}, a_{2}, a_{3}, \ldots, a_{n}, \ldots\right)$, where $a_{t}$ is the number of $q$ 's equal to the number $i$. We well-order the set of sequences of saying $\left(a_{1}, a_{2}, \ldots, a_{n}, \ldots\right)<\left(a_{1}^{\prime}, a_{2}^{\prime}, \ldots, a_{n}^{\prime}, \ldots\right)$ if and only if $a_{i}=a_{i}^{\prime}$, for $i>n$ and $a_{n}<a_{n}^{\prime}$.

Given any word $w$ it may have many representations of the form (*). We define the weight of the word $w$ to be the least weight of all the representations of $w$.

We shall see later, when dealing with transpositions, that a word need not have a unique representation of least weight.

REMARK 1. Let $w$ have representation $(*)$ of least weight.

(i) If $v_{i}=f\left(a_{i}\right) g\left(a_{i}\right)^{-1}$, then $t_{t, q_{i}} \neq f\left(a_{t}\right)^{-1}$ and $t_{i, q_{i}}^{-1} \neq g\left(a_{t}\right)$, since otherwise $w$ would have a representation of smaller weight.

(ii) No $t_{i, 1} \cdots t_{i, q_{t}} v_{i} t_{i, q_{t}}^{-1} \cdots t_{i, 1}^{-1}$ equals $x_{1} x_{2} \cdots x_{r}$ where each $x_{J}=$ $s_{j, 1} \cdots s_{j, n} f\left(b_{j}\right) g\left(b_{j}\right)^{-1} s_{j, n_{j}}^{-1} \cdots s_{n, 1}^{-1}$, where each $s_{l}$ lies in $F$ or $G$ and $s_{i}$ and $s_{i+1}$ do not both belong to $F$ or both belong to $G$ and $b_{j} \in A$, for $n_{j}<q_{l}, j=1,2, \ldots, r$. (This would contradict the fact that the representation of $w$ has least weight.)

(iii) If

$$
\begin{gathered}
\left(t_{l, 1} \cdots t_{l, q_{t}} v_{i} t_{i, q_{t}}^{-1} \cdots t_{i, 1}^{-1}\right)\left(t_{(i+1), 1} \cdots t_{(i+1), q_{t+1}} v_{i+1} t_{(l+1), q_{l+1}} \cdots t_{(l+1), 1}^{-1}\right) \\
\cdots\left(t_{(l+h), 1} \cdots t_{(t+h), q_{t+h}} v_{l+h} t_{(l+h), q_{l+h}}^{-1} \cdots t_{(l+h), 1}^{-1}\right) \\
=s_{1} \cdots s_{q} v s_{q}^{-1} \cdots s_{1}^{-1},
\end{gathered}
$$

where $v=f(a) g(a)^{-1}, a \in A$, and each $s_{i} \in F$ or $G$ then $q>$ $\max \left\{q_{l}, q_{i+1}, \ldots, q_{i+h}\right\}$.

(iv) Let

$$
x_{t}=t_{t, 1} \cdots t_{i, q_{t}} g\left(a_{i}\right) f\left(a_{i}\right)^{-1} t_{i, q_{i}}^{-1} \cdots t_{t, 1}^{-1} .
$$

Put $a=a_{i}^{-1}$. If $t_{t, q_{t}} \in F$, put $t=t_{i, q_{t}} f\left(a_{i}\right)$, so $t \in F$. If $t_{l, q_{t}} \in G$, put $t=t_{i, q_{t}} g\left(a_{l}\right)$, so $t \in G$. Then in both cases

$$
x_{\imath}=t_{i, 1} \cdots t_{i, q_{t-1}} t f(a) g(a)^{-1} t_{i, q_{t-1}}^{-1} \cdots t_{i, 1}^{-1} .
$$


The above rewriting shows that we can assume that if $(*)$ is a representation of a word $w \in K$, then each $v_{i}$ is of the form $f\left(a_{i}\right) g\left(a_{i}\right)^{-1}$. Because, if it is not, we can change the representation so that it is. Further, the change of representation does not affect the weight. So each $w \in K$ has a representation of the form (*) of least weight, with each $v_{i}=f\left(a_{i}\right) g\left(a_{i}\right)^{-1}$, for some $a_{i} \in A$.

Notation. From now on whenever we say we have a representation of the form $(*)$ we mean that $v_{i}=f\left(a_{i}\right) g\left(a_{i}\right)^{-1}$, for each $i$.

We need some further restrictions on the representations we consider.

DeFinitions. Let $x_{1}=t_{1,1} \cdots t_{1, q_{1}} f\left(a_{1}\right) g\left(a_{1}\right)^{-1} t_{1, q_{1}}^{-1} \cdots t_{1,1}^{-1}$ and $x_{2}$ $=t_{2,1} \cdots t_{2, q_{2}} f\left(a_{2}\right) g\left(a_{2}\right)^{-1} t_{2, q_{2}}^{-1} \cdots t_{2,1}^{-1}$.

(i) Let $q_{1} \geq q_{2}$ and $t_{1, j}=t_{2, j}$ for $1 \leq j \leq q_{2}-1$ and $t_{1, q_{2}}^{-1} t_{2, q_{2}} f\left(a_{2}\right)=$ $e$. If $q_{1}=q_{2}$ then $x_{1}$ is said to have an even link with $x_{2}$. If $q_{1} \neq q_{2}$ then $x_{1}$ is said to have an odd link with $x_{2}$.

(ii) Let $q_{1} \leq q_{2}$ and $t_{1, j}=t_{2, j}$ for $1 \leq j \leq q-1$ and $g\left(a_{1}\right)^{-1} t_{1, q_{1}}^{-1} t_{2, q_{1}}$ $=e$. If $q_{1}=q_{2}$ then $x_{1}$ is said to have an even link with $x_{2}$. If $q_{1} \neq q_{2}$ then $x_{1}$ is said to have an odd link with $x_{2}$.

(iii) If $x_{1}$ does not have an even link with $x_{2}$ or an odd link with $x_{2}$, then $x_{1}$ is said to have a neutral link with $x_{2}$.

(iv) A representation $x_{1} x_{2} \cdots x_{s}$ for $w \in K$, where each $x_{i}=t_{i, 1} \cdots$ $t_{i, q_{i}} v_{i} t_{i, q_{i}}^{-1} \cdots t_{i, 1}^{-1}$, for $v_{i}=f\left(a_{i}\right) g\left(a_{i}\right)^{-1}$, is said to be yoffi $i^{1}$ if it has least weight and each $x_{i}$ has a neutral link with $x_{i+1}$.

LEMMA 1. Let the representation (*) of $w \in K$ have least weight and be such that $q_{i}=q_{i+1}$ for some $i$. If $t_{i, j}=t_{i+1, j}, j=1, \ldots,\left(q_{i}-1\right), v_{i}=$ $f\left(a_{i}\right) g\left(a_{i}\right)^{-1}$ and $v_{i+1}=f\left(a_{i+1}\right) g\left(a_{i+1}\right)^{-1}$ then $t_{i, q_{i}}^{-1} t_{i+1, q_{t+1}} f\left(a_{i+1}\right) \neq e$ and $g\left(a_{i}\right)^{-1} t_{i, q_{t}}^{-1} t_{i+1, q_{i+1}} \neq e$; that is, $x_{i}$ does not have an even link with $x_{i+1}$.

Proof. Suppose $g\left(a_{i}\right)^{-1} t_{i, q_{t}}^{-1} t_{i+1, q_{t+1}}=e$. Then

$$
\left(t_{i+1, q_{t+1}}\right)^{-1}=g\left(a_{i}\right)^{-1} t_{i, q_{i}}^{-1} .
$$

So $\left[t_{i, 1} \cdots t_{i, q_{i}} v_{i} t_{i, q_{i}}^{-1} \cdots t_{i, 1}^{-1}\right]$.

${ }^{1} \mathrm{~A}$ Hebrew word meaning nice. 


$$
\begin{aligned}
& {\left[t_{(i+1), 1} \cdots t_{i+1, q_{i+1}} v_{i+1} t_{i+1, q_{t+1}}^{-1} \cdots t_{(i+1), 1}^{-1}\right]} \\
& \quad=t_{i, 1} \cdots t_{i, q_{t}} f\left(a_{i}\right)\left[g\left(a_{i}\right)^{-1} t_{i, q_{t}}^{-1} t_{i+1, q_{t}+1}\right] f\left(a_{i+1}\right) g\left(a_{i+1}\right)^{-1} t_{i+1, q_{i+1}}^{-1} \\
& \quad \cdots t_{i+1,1}^{-1} \\
& \quad=t_{i, 1} \cdots t_{i, q_{t}} f\left(a_{i}\right) f\left(a_{i+1}\right) g\left(a_{i+1}\right)^{-1} t_{i+1, q_{t+1}}^{-1} \cdots t_{(i+1), 1}^{-1} \\
& =t_{i, 1} \cdots t_{i, q_{t}} f\left(a_{i}\right) f\left(a_{i+1}\right) g\left(a_{i+1}\right)^{-1}\left[g\left(a_{i}\right)^{-1} t_{i, q_{t}}^{-1}\right] \cdots t_{(i+1), 1}^{-1} \\
& =t_{i, 1} \cdots t_{i, q_{i}} f\left(a_{i} a_{i+1}\right) g\left(a_{i} a_{i+1}\right)^{-1} t_{i, q_{i}}^{-1} \cdots t_{(i+1), 1}^{-1} .
\end{aligned}
$$

This is clearly a contradiction to $(*)$ having least weight. So $g\left(a_{i}\right)^{-1} t_{i, q_{t}}^{-1} t_{i+1, q_{t+1}} \neq e$. Similarly it can be shown that $t_{i, q_{t}}^{-1} t_{i+1, q_{t+1}} f\left(a_{i+1}\right)$ $\neq e$.

Notation. Let $X=\cup_{n=1}^{\infty}\left\{u v u^{-1}: u \in(F \cup G)^{n}, v=f(a) g(a)^{-1}\right.$, $a \in A\}$, where $(F \cup G)^{n}$ denotes the set of words which are the product of at most $n$ elements from $F \cup G$.

LEMMA 2. Let $x_{1}=t_{1,1} \cdots t_{1, q_{1}} f\left(a_{1}\right) g\left(a_{1}\right)^{-1} t_{1, q_{1}}^{-1} \cdots t_{1,1}^{-1}$ and $x_{2}=$ $t_{2,1} \cdots t_{2, q_{2}} f\left(a_{2}\right) g\left(a_{2}\right)^{-1} t_{2, q_{2}}^{-1} \cdots t_{2,1}^{-1}$ be in $X$. If $x_{1}$ has an odd link with $x_{2}$ then $x_{1} x_{2}=x_{2}^{\prime} x_{1}^{\prime}$, where $x_{2}^{\prime}$ and $x_{1}^{\prime}$ are in $X$, the weight of the representation $x_{1} x_{2}$ equals the weight of the representation $x_{2}^{\prime}, x_{1}^{\prime}$, and $x_{2}^{\prime}$ has a neutral link with $x_{1}^{\prime}$.

Proof. Without loss of generality, assume $q_{1}>q_{2}$. Then $t_{1, j}=t_{2, j}$ for $1 \leq j \leq q_{2}-1$ and $t_{1, q_{2}}^{-1} t_{2, q_{2}} f\left(a_{2}\right)=e$. Put

$$
x_{2}^{\prime}=t_{2,1} \cdots t_{2, q_{2}} f\left(a_{2}\right) g\left(a_{2}\right)^{-1} t_{2, q_{2}}^{-1} \cdots t_{2,1}^{-1} \text {, }
$$

and

$$
\begin{array}{r}
x_{1}^{\prime}=\left[t_{2,1} \cdots t_{2, q_{2}}\left(g\left(a_{2}\right) t_{1, q_{2}+1}\right) t_{1, q_{2}+2} \cdots t_{1, q_{1}} f\left(a_{1}\right) g\left(a_{1}\right)^{-1} t_{1, q_{1}}^{-1}\right. \\
\left.\cdots t_{1, q_{2}+2}^{-1}\left(t_{1, q_{2}+1}^{-1} g\left(a_{2}\right)^{-1}\right) t_{2, q_{2}}^{-1} \cdots t_{2,1}^{-1}\right] .
\end{array}
$$

Then $x_{2}^{\prime} x_{1}^{\prime}$ has the required properties.

Definition. The change of representation from $x_{1} x_{2}$ to $x_{2}^{\prime} x_{1}^{\prime}$, when $q_{1}>q_{2}$ or when $q_{2}>q_{1}$, is called a transposition.

Proposition 1. Each $w \in K$ has a yoffi representation. 
Proof. Choose a representation of $w$ of the form (*) which has least weight. Let this representation be $w=x_{1} \cdots x_{s}$, where $x_{i}=t_{l, 1} \cdots$ $t_{i, q_{i}} v_{i} t_{i, q_{i}}^{-1} \cdots t_{i, 1}^{-1}$ where $v_{i}=f\left(a_{i}\right) g\left(a_{i}\right)^{-1}$ for $i=1, \ldots, s$.

Our proof will be by induction on $s$. Firstly consider the case $s=2$; that is, $w=x_{1} \cdot x_{2}$. If $x_{1}$ has a neutral link with $x_{2}$, then this is a yoffi representation. Observe that by Lemma $1, x_{1}$ cannot have an even link with $x_{2}$. So we let $x_{1}$ have an odd link with $x_{2}$. Then by Lemma 2 we can apply a transposition to obtain $x_{2}^{\prime} x_{1}^{\prime}$ such that this representation also has least weight and $x_{2}^{\prime}$ has a neutral link with $x_{1}^{\prime}$. Hence $x_{2}^{\prime} x_{1}^{\prime}$ is a yoffi representation of $w$.

Now suppose that every word of the form $w=x_{1} x_{2} \cdots x_{n}$, for $n \leq s$, has a yoffi representation. Then consider any word

$$
w=x_{1} x_{2} \cdots x_{s+1}
$$

where the weight of the word $w$ equals the weight of the representation $x_{1} \cdots x_{s+1}$. Clearly the weight of the word $x_{1} x_{2} \cdots x_{s}$ equals the weight of the representations $x_{1} \cdots x_{s}$. Thus by the inductive hypothesis it has a yoffi representation of the same weight. So without loss of generality we can assume that it is $x_{1} \cdots x_{s}$. So each $x_{i}$ has a neutral link with $x_{i+1}$ for $i=1, \ldots, s-1$. If $x_{s}$ has a neutral link with $x_{s+1}$ we are done. If not, we perform a transposition of $x_{s} x_{s+1}$ to obtain from $w$ the representation $x_{1} x_{2} \cdots x_{s-1} x_{s+1}^{\prime} x_{s}^{\prime}$. If $x_{s-1}$ has a neutral link with $x_{s+1}^{\prime}$, we are done. If $x_{s-1}$ has an odd link with $x_{s+1}^{\prime}$ then we apply a transposition of $x_{s-1} x_{s+1}^{\prime}$ to obtain the representation $x_{1} x_{2} \cdots x_{s-2} x_{s+1}^{\prime \prime} x_{s-1}^{\prime} x_{s}^{\prime}$. By Lemma 3 (proved independently below) $x_{s-1}^{\prime} x_{s}^{\prime}$ has a neutral link. Once again, if $x_{s-2} x_{s+1}^{\prime \prime}$ has a neutral link we are done. If not, apply a transposition of $x_{s-2} x_{s+1}^{\prime \prime}$ to obtain $x_{1} x_{2} \cdots x_{s-3} x_{s+1}^{\prime \prime \prime} x_{s-2}^{\prime} x_{s-1}^{\prime} x_{s}^{\prime}$. By Lemma 3 again $x_{s-2}^{\prime} x_{s}^{\prime}$ has a neutral link. Continuing this process, if necessary, we obtain a yoffi representation. So the proof is complete once we have Lemma 3.

LEMMA 3. Let $x_{1} x_{2} x_{3}$ be a representation of $a$ word $w$ such that the weight of the word $w$ equals the weight of the representation $x_{1} x_{2} x_{3}$. If $x_{1}$ has a neutral link with $x_{2}$, then $x_{1} x_{2} x_{3}, x_{1} x_{3}^{\prime} x_{2}^{\prime}$ or $x_{3}^{\prime \prime} x_{1}^{\prime} x_{2}^{\prime}$ is a yoffi representation where $x_{3}^{\prime} x_{2}^{\prime}$ is a transposition of $x_{2} x_{3}$ and $x_{3}^{\prime \prime} x_{1}^{\prime}$ is a transposition of $x_{1} x_{3}^{\prime}$.

Proof. If $x_{1} x_{2} x_{3}$ is not a yoffi representation let

$$
x_{i}=t_{i, 1} \cdots t_{i, q_{i}} f\left(a_{i}\right) g\left(a_{i}\right)^{-1} t_{i, 1_{i}}^{-1} \cdots t_{i, 1}^{-1}, \quad \text { for } i=1,2,3 .
$$

We distinguish six cases:
(i) $q_{1}<q_{2}<q_{3}$,
(ii) $q_{1}<q_{3}<q_{2}$,
(iii) $q_{3}<q_{2}<q_{1}$,
(iv) $q_{2}<q_{3}<q_{1}$,
(v) $q_{3}<q_{1}<q_{2}$,
(vi) $q_{2}<q_{1}<q_{3}$. 
Consider case (i). We claim that $x_{1}$ has a neutral link with $x_{3}^{\prime}$ since $x_{1}$ has a neutral link with $x_{2}$ and the first $q_{1}$ elements of $x_{2}$ and $x_{3}^{\prime}$ are the same. Hence $x_{1} x_{3}^{\prime} x_{2}^{\prime}$ is a yoffi representation. Exactly the same argument works in case (ii). Now consider case (iii). If $x_{1}$ has a neutral link with $x_{3}^{\prime}$ we are done. If not, consider $x_{3}^{\prime \prime} x_{1}^{\prime} x_{2}^{\prime}$. We have to show that $x_{1}^{\prime}$ has a neutral link with $x_{2}^{\prime}$. In obtaining $x_{2}^{\prime}$ from $x_{2}$ we replace only $t_{2, q_{3}}$ by $t_{3, q_{3}}$ and $t_{2, q_{3}+1}$ by $g\left(a_{3}\right) t_{2, q_{3}+1}$ and in obtaining $x_{1}^{\prime}$ from $x_{1}$ we replace only $t_{1, q_{3}}$ by $t_{3, q_{3}}$ and $t_{1, q_{3}+1}$ by $g\left(a_{3}\right) t_{1, q_{3}+1}$, because $x_{3}=x_{3}^{\prime}$ in this case. The fact that $x_{1}$ has a neutral link with $x_{2}$ then clearly implies that $x_{1}^{\prime}$ has a neutral link with $x_{2}^{\prime}$. Hence $x_{3}^{\prime \prime} x_{1}^{\prime} x_{2}^{\prime}$ is a yoffi representation.

Cases (iv)-(vi) are proved in the same fashion.

Finally observe that $q_{2}=q_{3}$ implies that $x_{2}$ has a neutral link with $x_{3}$ and so $x_{1} x_{2} x_{3}$ is a yoffi representation, $q_{1}=q_{2}$ implies $x_{3}^{\prime \prime} x_{1}^{\prime} x_{2}^{\prime}$ is a yoffi representation, and $q_{1}=q_{3}$ implies $x_{1} x_{3}^{\prime} x_{2}^{\prime}$ is a yoffi representation. This completes the proof of the lemma.

4. The reduced lengths of elements of $K$. The key results of this section are Propositions 2 and 3 which imply that if $w=x_{1} x_{2} \cdots x_{s}$ is a yoffi representation and $w$ has reduced length $\leq n$ (that is, $\left.w \in(F \cup G)^{n}\right)$, then $s \leq n$ and $2 q_{i}+1 \leq 4 n$, for $i=1,2, \ldots, s$.

LEMMA 4. Let $w \in K$ have representation (*) of least weight. If $q_{i}>q_{i+1}$ for some $i$ and $t_{i, j}=t_{i+1, j}$, for $j=1, \ldots, q_{i+1}$, then $t_{i\left(q_{i+1}+1\right)} \neq f\left(a_{i+1}\right)$.

Proof. Suppose $t_{i\left(q_{i+1}+1\right)}=f\left(a_{i+1}\right)$. Then

$$
\begin{gathered}
t_{i, 1} \cdots t_{i, q_{i}} v_{i} t_{i, q_{i}}^{-1} \cdots t_{i, 1}^{-1} \\
=\left(t_{i, 1} \cdots t_{i, q_{i+1}} f\left(a_{i+1}\right) g\left(a_{i+1}\right)^{-1} t_{i, q_{i+1}}^{-1} \cdots t_{i, 1}^{-1}\right) \\
\cdot\left(t_{i, 1} \cdots\left[t_{i, q_{i+1}} g\left(a_{i+1}\right) t_{i\left(q_{i+1}+2\right)}\right] \cdots t_{i, q_{l}} v_{i} t_{i, q_{i}}^{-1}\right. \\
\left.\cdots\left[t_{i, q_{i+1}+2}^{-1} g\left(a_{i+1}\right)^{-1} t_{i, q_{i+1}+1}\right] \cdots t_{i, 1}^{-1}\right) \\
\cdot\left(t_{i, 1} \cdots t_{i, q_{i+1}} g\left(a_{i+1}\right) f\left(a_{i+1}\right)^{-1} t_{i, q_{i+1}}^{-1} \cdots t_{i, 1}^{-1}\right) .
\end{gathered}
$$

As the weight of the representation on the left side of the equality is greater than the weight of the representation on the right side, we have a contradiction. Hence $t_{i, q_{i+1}+1} \neq f\left(a_{i+1}\right)$. 
Proposition 2. Let $w \in K$ and have yoffi representation (*). Then for each $i \in\{1,2, \ldots, s\}, g\left(a_{i}\right)^{-1} t_{i, q_{i}}^{-1} \cdots t_{i, 1}^{-1} t_{i+1,1} \cdots t_{i+1, q_{i+1}} f\left(a_{i+1}\right)$ in reduced form equals $g_{1} f_{1} g_{2} f_{2} \cdots f_{k_{i}}$ where each $f_{j} \in F \backslash\{e\}, g_{j} \in G \backslash\{e\}$, and $k_{i} \geq 1$.

Proof. It suffices to show that in putting the relevant word in reduced form, the elements $g\left(a_{i}\right)^{-1}$ and $f\left(a_{i+1}\right)$ do not get cancelled. Indeed, we prove only that $f\left(a_{i+1}\right)$ does not get cancelled, as the other follows by an analogous argument. The element $f\left(a_{i+1}\right)$ could get cancelled out only in one of the following ways; but all are impossible, as indicated by the bracketed remarks.

(1) $t_{i+1, q_{t+1}}^{-1}=f\left(a_{i+1}\right) \quad($ Remark 1(i))

(2) $f\left(a_{i+1}\right)=t_{i, q_{t+1}+1}$ and

$$
t_{i, j}=t_{(i+1), j}, \quad j=1, \ldots, q_{i+1} \text {. }
$$

$$
\begin{aligned}
& t_{i, q_{i+1}}^{-1} t_{(i+1), q_{i+1}} f\left(a_{i+1}\right)=e \quad \text { and } \\
& t_{i, j}=t_{(i+1), j}, \quad j=1, \ldots, q_{i+1}-1 \quad \text { (yoffi implies neutral link). }
\end{aligned}
$$

Thus $f\left(a_{i+1}\right)$ does not get cancelled out. So we see that the proposition is true.

Remark 2. Proposition 2 implies that if $w=x_{1} \cdots x_{s}$ is a yoffi representation and has reduced length $\leq n$, then $s \leq n$.

LEMMA 5. Let $w \in K$ have yoffi representation (*). If the reduced length of $w^{\prime}=t_{1,1} \cdots t_{1, q_{1}} v_{1} \cdots v_{s-1} t_{(s-1), q_{s-1}}^{-1} \cdots t_{(s-1), 1}^{-1}$ is $m$, then the reduced length of $w$ is greatre than or equal to $m-3$.

Proof. By Proposition 2, in order to obtain the reduced form of $w$ from the reduced form of $w^{\prime}$ it suffices to ascertain what is the reduced form of

$$
g\left(a_{s-1}\right)^{-1} t_{s-1, q_{s-1}}^{-1} \cdots t_{s-1,1}^{-1} t_{s, 1} \cdots t_{s, q_{s}} f\left(a_{s}\right) g\left(a_{s}\right)^{-1} t_{s, q_{s}}^{-1} \cdots t_{s, 1}^{-1} .
$$

From what we have said earlier it is obvious that the reduced length of $g\left(a_{s-1}\right)^{-1} t_{s-1, q_{s-1}}^{-1} \cdots t_{s, q_{s}} f\left(a_{s}\right)$ is greater than or equal to $\left|q_{s-1}-q_{s}\right|-2$. The reduced length of $g\left(a_{s}\right) t_{s, q_{s}}^{-1} \cdots t_{s, 1}^{-1}$ is greater than or equal to $q_{s}$.

Noting that the length of $t_{1,1} \cdots v_{1} \cdots f\left(a_{s-1}\right)$ is greater than or equal to $m-\left(q_{s-1}+1\right)$, we see that the reduced length of $w$ is greater than or equal to $m-q_{s-1}-1+\left|q_{s-1}-q_{s}\right|-2+q_{s} \geq m-3$. 
REMARK 3. Of course the bound $m-3$ in Lemma 5 is a crude one, but it serves the purpose.

Proposition 3. Let $w \in K$ have yoffi representation $(*)$. If the reduced length of $w$ is $n$ then $2 q_{i}+1 \leq n+3 s$, for each $i=1, \ldots, s$.

Proof. Consider $t_{i, 1} \cdots v_{i} \cdots t_{i, 1}^{-1}$. Successively multiplying on the right or left with words of the same kind, we obtain the word $w$. In so doing, we can apply Lemma 5 above $s-1$ times, and the result follows.

Remark 4. Combining Remark 2 and Proposition 3 we obtain the desired result $2 q_{i}+1 \leq 4 n$.

5. Proof of the Theorem. To prove the main result, which is the theorem stated in $\$ 2$, we must now study the $k_{\omega}$ structure.

It is known [12] that the free product of two $k_{\omega}$-groups is a $k_{\omega}$-group. More particularly, if $F$ and $G$ have $k_{\omega}$ decompositions $F=\bigcup_{n} F_{n}$ and $G=\cup_{n} G_{n}$ then the free product has $k_{\omega}$ decomposition

$$
F * G=\bigcup_{n}\left(F_{n} \cup G_{n}\right)^{n}
$$

For convenience, we assume $f(A) \subseteq F_{1}, g(A) \subseteq G_{1}, F_{n}=F_{n}^{-1}, G_{n}=G_{n}^{-1}$, $F_{n} F_{m} \subseteq F_{n+m}$ and $G_{n} G_{m} \subseteq G_{n+m}$.

Recall that $\Phi$ is the canonical continuous homomorphism $F * G \rightarrow$ $F *{ }_{A} G$.

Notation. Let

$$
\begin{aligned}
X_{n} & =\left\{u v u^{-1}: u \in\left(F_{n} \cup G_{n}\right)^{n}, v=f(a) g(a)^{-1}, a \in A\right\} \\
X & =\bigcup_{n=1}^{\infty} X_{n} \text { and } Y_{n}=\left(X_{n}\right)^{n} .
\end{aligned}
$$

Here $\left(X_{n}\right)^{n}$ denotes the set of all words which are the product of at most $n$ elements from $X_{n}$.

Clearly each $X_{n}$ and $Y_{n}$ is compact, and $K=\cup_{n=1}^{\infty} Y_{n}$.

REMARK 5. To prove that $K$ is closed, it suffices to show that for each $n$,

$$
K \cap\left(F_{n} \cup G_{n}\right)^{n} \subseteq Y_{2 n^{3}} .
$$

This suffices, since then we would have

$$
K \cap\left(F_{n} \cup G_{n}\right)^{n}=K \cap\left(F_{n} \cup G_{n}\right)^{n} \cap Y_{2 n^{3}}=Y_{2 n^{3}} \cap\left(F_{n} \cap G_{n}\right)^{n}
$$


which is compact, and hence we would have $K$ closed in $F * G$ by the $k_{\omega}$-condition.

Proposition 1 implies that each $w \in K \cap\left(F_{n} \cup G_{n}\right)^{n}$ has a yoffi representation (*). Remarks 2 and 4 then imply that $s \leq n$ and $2 q_{i}+1 \leq$ $4 n$, for each $i$. To complete the proof of (1) above (and hence of the theorem) it suffices to show that each $t_{i, j} \in F_{2 n^{3}} \cup G_{2 n^{3}}$. This is a consequence of Proposition 4, proved below.

Proposition 4. Let $w \in K$ have yoffi representation (*). If $w \in$ $\left(F_{n} \cup G_{n}\right)^{n}$, then each $t_{i, j} \in F_{n^{3}+n} \cup G_{n^{3}+n}$.

Proof. By Proposition 2, we know that $w$ in its reduced form is the product of the reduced form of the following blocks:

$$
\begin{aligned}
t_{1,1} \cdots t_{1, q_{1}} f\left(a_{1}\right) ; & g\left(a_{1}\right)^{-1} \cdots f\left(a_{2}\right) ; \quad g\left(a_{2}\right)^{-1} \cdots f\left(a_{3}\right) ; \ldots ; \\
& g\left(a_{s-1}\right)^{-1} \cdots f\left(a_{s}\right) ; \quad g\left(a_{s}\right)^{-1} t_{s, q_{s}}^{-1} \cdots t_{s, 1}^{-1} .
\end{aligned}
$$

The reduced form of $w$ in $F * G$ is unique, so each block in the reduced yoffi representation of $w$ matches a part of the reduced form obtained from the word $w \in\left(F_{n} \cup G_{n}\right)^{n}$. Thus any reduced block of $w$ can be expanded to part of a word in $\left(F_{n} \cup G_{n}\right)^{n}$ and is therefore itself in $\left(F_{n} \cup G_{n}\right)^{n}$.

Consider the block $B_{0}=t_{1,1} \cdots t_{1, q_{1}} f\left(a_{1}\right)$. Recalling that $f(A) \subseteq F_{1}$, $g(A) \subseteq G_{1}, F_{n} F_{m} \subseteq F_{n+m}$ and $G_{n} G_{m} \subseteq G_{n+m}$, and noting that each $t_{1, j}$ $(1 \leq j \leq q-1)$ can be expanded to a part of a word in $\left(F_{n} \cup G_{n}\right)^{n}$, at worst a product of $n$ elements all from $F_{n}$ (or $G_{n}$ ) it follows that $t_{1, j} \in F_{n^{2}} \cup G_{n^{2}}$. If $t_{1, q_{1}} \in F$, then, at worst $t_{1, q_{1}} f\left(a_{1}\right) \in F_{n^{2}}$, so $t_{1, q_{1}} \in F_{n^{2}+1}$. Therefore $t_{1, j} \in F_{n^{2}+1} \cup G_{n^{2}+1}$, for $j=1, \ldots, q_{1}$.

Consider the block $B_{1}=g\left(a_{1}\right)^{-1} \cdots f\left(a_{2}\right)$. Each element in $B_{1}$ is also in the reduced form (of $B_{1}$ ) or is amalgamated by other elements of $B_{1}$. Such an amalgamation can involve at most three elements. At most one of these three is a $t_{2, j}$. Of the others at most one is a $t_{1, k}$, and the other is in $f(A) \cup g(A)$. As the elements of the reduced form of $w$ lie in $F_{n^{2}} \cup G_{n^{2}}$, we see that each element of $B_{1}$ must lie in $F_{2 n^{2}+2} \cup G_{2 n^{2}+2}$.

We now proceed to consider in a similar fashion $B_{2}, B_{3}, \ldots, B_{s-1}$. We thus obtain that each $t_{i, j}$ that appears in $w$ is an element of $F_{s\left(n^{2}+1\right)} U$ $G_{s\left(n^{2}+1\right)}$. The result now follows by observing that $s \leq n$.

REMARK 6. To prove the theorem, it suffices to show that the kernel $K$ of $\Phi$ is a closed subgroup of $F * G$. Observing that $\Phi(F) \cap \Phi\left(\left(F_{n} \cup G_{n}\right)^{n}\right)$ $\subseteq \Phi\left(\left[F_{n^{2}} \cup\left(G_{n^{2}} \cap A\right)\right]^{n}\right)$ it then follows that $F$ is a closed topological 
subgroup of $F *{ }_{A} G$. Similarly $G$ is seen to be a closed topological subgroup of $F *{ }_{A} G$ also.

We have thus completed the proof of the theorem.

REMARK 7. We conclude by noting that if $G$ is any connected locally compact group and $K$ is a maximal compact subgroup, then the amalgamated free product $G{ }^{*}{ }_{K} G$ is seen, from our Theorem, to be a $k_{\omega}$-group (and hence Hausdorff). This example could not be deduced from Ordman [13], Khan and Morris [5] or Katz and Morris [4].

REMARK 8. Our Theorem includes the case when $K$ is a finite group. It should be interesting to investigate this case further.

\section{REFERENCES}

[1] T. H. Fay and B. V. S. Thomas, Remarks on the free product of two Hausdorff groups, Arch. Math. (Basel), 33 (1979), 57-65.

[2] M. I. Graev, On free products of topological groups, (Russian), Izv. Akad. Nauk. SSSR, Ser. Mat., 14 (1950), 343-354.

[3] E. Katz, Free products in the category of $k_{\omega}$-groups, Pacific J. Math., 59 (1975), 493-495.

[4] E. Katz and S. A. Morris, Free products of $k_{\omega}$-topological groups with normal amalgamation, Topology Appl., 15 (1983), 189-196.

[5] M. S. Khan and S. A. Morris, Free products of topological groups with central amalgamation I, Trans. Amer. Math. Soc., 273 (1982), 417-432.

[6] W. F. La Martin, On the foundations of $k$-group theory, Dissertationes mathematicae \#146 (1977).

[7] W. Magnus, A. Karrass and D. Solitar, Combinatorial Group Theory, (Dover Pub1. Inc., New York, 1976).

[8] S. A. Morris, E. T. Ordman and H. B. Thompson, The Topology of Free Products of Topological Groups, Proc. Second Internat. Conf. Theory of Groups, Canberra (1973), Springer Lecture Notes 372, 504-515.

[9] B. H. Neumann, an essay on free products of groups with amalgamation, Philos. Trans. Royal Soc. London (A), 246 (1954), 503-554.

[10] P. Nickolas, A Kurosh subgroup theorem for topological groups, Proc. London Math. Soc., 42 (81), 461-477.

[11] E. C. Nummela, On epimorphisms of topological groups, General Topology Appl., 9 (1978), 155-167.

[12] E. T. Ordman, Free products of topological groups which are $k_{\omega}$-spaces, Trans. Amer. Math. Soc., 191 (1974), 61-73.

[13] , Free products of topological groups with equal uniformities I, Colloq. Math., 31 (1974), 37-43.

[14] B. V. S. Thomas, Categories of topological groups, Memphis State University Report 76-16.

[15] On the Coproduct of the Topological Groups $Q$ and $Z_{2}$, Proc. Int. Conf. on categorical topology, Berlin, 1978. Springer Lecture Notes, 719 (1979), 371-375. 
Received July 21, 1980 and in revised form July 4, 1984. Some of the research for this paper was carried out while the authors were visiting Tel-Aviv University. The first author thanks La Trobe University for hospitality extended in June to August 1981, and the second author thanks Cleveland State University for hospitality extended in August 1983. The authors gratefully acknowledge the most helpful comments of the referees.

Cleveland State University

Cleveland, OH 44115

AND

LA TROBE UNIVERSITY

BUNDOORA, VICTORIA

3083, Australia 


\section{PACIFIC JOURNAL OF MATHEMATICS EDITORS}

\author{
V. S. VARADARAJAN (Managing Editor) \\ University of California \\ Los Angeles, CA 90024 \\ Charles R. DePrima \\ California Institute of Technology \\ Pasadena, CA 91125 \\ R. FINN \\ Stanford University \\ Stanford, CA 94305
}

\author{
HeRmanN FlaschKa \\ University of Arizona \\ Tucson, AZ 85721 \\ Ramesh A. Gangolli \\ University of Washington \\ Seattle, WA 98195 \\ ROBION KIRBY \\ University of California \\ Berkeley, CA 94720
}

C. C. MOORE

University of California

Berkeley, CA 94720

H. SAMELSON

Stanford University

Stanford, CA 94305

HAROLD STARK

University of California, San Diego

La Jolla, CA 92093

ASSOCIATE EDITORS
R. ARENS
E. F. BECKENBACH
B. H. NeUmanN
F. WOLF
K. YosHIDA (1906-1982)

\section{SUPPORTING INSTITUTIONS}

UNIVERSITY OF ARIZONA

UNIVERSITY OF BRITISH COLUMBIA

CALIFORNIA INSTITUTE OF TECHNOLOGY

UNIVERSITY OF CALIFORNIA

MONTANA STATE UNIVERSITY

UNIVERSITY OF NEVADA, RENO

NEW MEXICO STATE UNIVERSITY

OREGON STATE UNIVERSITY
UNIVERSITY OF OREGON

UNIVERSITY OF SOUTHERN CALIFORNIA

STANFORD UNIVERSITY

UNIVERSITY OF HAWAII

UNIVERSITY OF TOKYO

UNIVERSITY OF UTAH

WASHINGTON STATE UNIVERSITY

UNIVERSITY OF WASHINGTON 


\section{Pacific Journal of Mathematics}

Vol. 119, No. $1 \quad$ May, 1985

Maurice Chacron, Nonisotropic unitary spaces and modules with

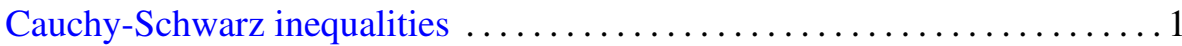

Myriam Dechamps-Gondim, Françoise Piquard and H. Queffélec, On

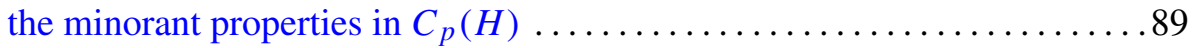

Klaus Floret and V. B. Moscatelli, On bases in strict inductive and projective limits of locally convex spaces .................... 103

Norman Joseph Goldstein, Degenerate secant varieties and a problem on

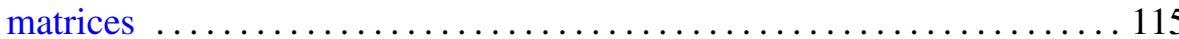

Harold Morris Hastings and Stefan Waner, $G$-bordism with singularities

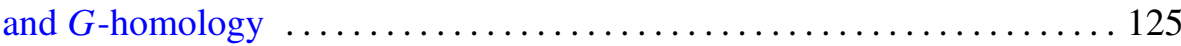

Takesi Isiwata, Clopen realcompactification of a mapping ........... 153

Hisao Kato, Concerning hyperspaces of certain Peano continua and strong

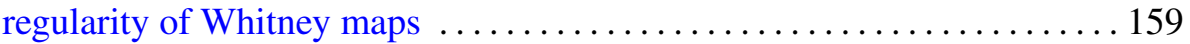

Elyahu Katz and Sidney Allen Morris, Free products of topological groups

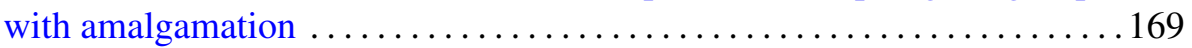

Kyewon Koh Park, Nice dense subsets for ergodic flows and Bernoulli

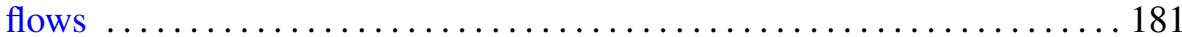

Francis Pastijn and Peter George Trotter, Lattices of completely regular

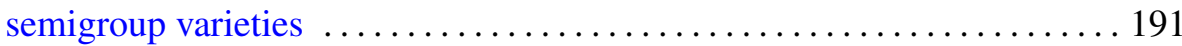

Rae Michael Andrew Shortt, Reticulated sets and the isomorphism of analytic powers

David A. Stegenga and Kenneth R. Stephenson, Generic covering properties for spaces of analytic functions

M. V. Subba Rao and R. Sitaramachandra Rao, On some infinite series of

L. J. Mordell and their analogues 